\title{
Cuestionario de Bienestar Laboral General: Estructura y Propiedades Psicométricas
}

\section{Questionnaire of General Labor Well-Being: Structure and Psychometric Properties}

\author{
Josep M. Blanch, Miguel Sahagún, Leonor Cantera y Genís Cervantes \\ Universidad Autónoma de Barcelona
}

\begin{abstract}
Resumen. A un colectivo de 1252 profesionales de la sanidad (medicina y enfermería) y de la educación públicas que trabajan respectivamente en hospitales y en universidades de Brasil, Chile, Colombia y España, se le ha aplicado el Cuestionario de Bienestar Laboral General (qBLG) que incluye series de ítems cerrados organizados de acuerdo con un modelo teórico según el cual el bienestar laboral general (qBLG) incluye una dimensión de bienestar psicosocial (integrada por afectos, competencias y expectativas) y otra de efectos colaterales (somatización, desgaste y alienación). Un análisis de componentes principales mostró la existencia de dos factores independientes en el qBLG, incluyendo el primero de ellos tres escalas de bienestar psicosocial y el segundo otras tres de efectos colaterales. Todas las escalas mostraron una alta consistencia interna, con valores $\alpha$ de Cronbach comprendidos entre .82 (alienación) y .96 (expectativas). Los resultados indican que el cuestionario BLG reproduce fielmente la estructura del modelo teórico propuesto.
\end{abstract}

Palabras clave: bienestar psicosocial, somatización, estructura factorial, fiabilidad, cuestionario.

\begin{abstract}
The general work well-being questionnaire (qBLG) was developed with the aim of providing a measurement tool particularly sensitive to the psychosocial dimension of work well-being of care workers, and to the bipolar nature of the well-being experience. 1252 professionals of health care (medical and nursing) and of higher education employed in hospitals and universities from Brazil, Chile, Colombia and Spain, respectively, answered a series of closed items from a questionnaire organized according with a theoretical model of general work well-being. Factor analysis found two independent factors in the qBLG, the first one including three scales of psychosocial well-being and the second three scales of collateral effects. All scales showed high internal consistency. The qBLG faithfully reproduces the structure of the proposed theoretical model, and combines the required psychometric properties for the evaluation of work well-being of health care and of higher education professionals.

Key words: psychosocial well-being, somatization, factor structure, reliability, questionnaire.
\end{abstract}

El bienestar constituye un referente teórico de primer orden, especialmente a partir del momento en que la Organización Mundial de la Salud lo utiliza, en su declaración constitucional de 1948, como piedra angular de su definición de salud como "estado de completo bienestar físico, psicológico y social". Desde entonces, las complejas e intensas relaciones entre bienestar y trabajo han centrado el interés de la psicología en general y particularmente el de la psicología del trabajo y de las organizaciones. En este contexto, términos como los de bienestar, felicidad, satisfacción, salud, e incluso optimismo, flow y expresiones como las de salud mental, calidad de vida o experiencia óptima han sido usados unas veces como sinónimos e intercambiables (Avia y Vázquez, 1998; Blanco y Díaz, 2005; Csikszentmihalyi, 1990; Hills y Argyle, 2002;

La investigación incluida en este artículo fue parcialmente financiada por el proyecto SEJ2007-63686/PSIC. La correspondencia deberá enviarse a Josep M. Blanch, Departament Psicologia Social, Universitat Autònoma de Barcelona, Campus UAB, 08193, Bellaterra, Barcelona. E-mail: josepmaria.blanch@uab.cat
Seligman, 2002; Seligman y Csikszentmihalyi, 2000; Veenhoven, 1984; 1994; 1995; Warr, 1990) y otras para invocar significados diferenciados; si bien como pertenecientes a un mismo campo semántico (Morrison et al, 2005; Shaw y Gupta, 2004; Warr, 2007; White, 2007). Lo mismo puede decirse a propósito de expresiones como las de bienestar subjetivo (Strack, Argile y Schwartz, 1991; Diener, 2000; Keyes et al, 2002) y bienestar psicológico (Bryce y Hawort, 2003; Ryff y Keyes, 1995, Warr, 1987; 1990). Según la acepción más comúnmente aceptada, el bienestar (subjetivo o psicológico) consiste en un conjunto de juicios valorativos y de reacciones emocionales concernientes al grado en que la propia experiencia es vivida como satisfactoria, agradable y positiva (Andrews y Withey, 1976; Diener, 2000; Diener y Suh, 2001; Diener, Oishi y Lucas, 2003; Lucas, Diener y Suh, 1996).

En la experiencia de estar bien en la vida y en el trabajo confluyen factores situacionales y personales. De ahí deriva la coexistencia, más o menos tensa, de explicaciones "situation centered" y "person centered", en 
un el juego dialéctico marcado por el predominio del segundo enfoque, a través del cual la persona trabajadora aparece no sólo como el escenario de manifestación del bienestar laboral, sino también como un decisivo factor de producción del mismo. Desde esta óptica, el estado de bienestar laboral es tratado generalmente como consecuencia ("effect of", "reaction to", etc.) personal de algún input organizacional -"working conditions", "job change", etc.- (De Cuyper, Isaksson y De Witte, 2005; De Witte, 1999; Warr, 1987; 2007) y excepcionalmente como factor causante de determinadas consecuencias personales $\mathrm{u}$ organizacionales (Lucas y Diener, 2003).

Esta reducción del foco de atención al nivel individual constituye una manifestación de la hegemonía de la mirada clínica que, a su vez, refuerza el sesgo patologista de la psicología del bienestar en el trabajo, centrada más bien en el análisis del malestar laboral. Según Schaufeli y Backer (2004), hasta principios del siglo XXI, las investigaciones psicológicas sobre aspectos negativos de la experiencia laboral (distrés, burnout, ansiedad, depresión, trastornos mentales menores, disfunciones psicofisiológicas, etc.) predominaron con un ratio de 15 a 1 sobre las que trataron de facetas positivas de la misma (felicidad, satisfacción, bienestar, autorrealización, etc.). Cuando se piensa de este modo las causas y las consecuencias del bienestar laboral en la persona trabajadora, se tiende a buscar prioritariamente "síntomas" individuales. Así, se relega la información sobre las características organizacionales del trabajo al apartado de los datos censales; lo que de paso les confiere el estatus de realidades casi naturales, como el sexo, la edad o la antigüedad en el puesto. Lo cual inspira, a su vez, el diseño de la investigación y el formato de los instrumentos de evaluación, que cumplen por ello una doble función epistemológica sobreañadida: (a) tautológica, induciendo al descubrimiento en forma de resultados empíricos de unos supuestos previamente asumidos sobre la realidad del objeto y (b) prescriptiva, imponiendo un determinado nivel de análisis y una particular selección de las características definidas como relevantes del fenómeno. ¿Cómo se explica, si no, el que entre las herramientas más frecuentemente utilizadas para evaluar el bienestar laboral destaque el General Health Questionnaire $(G H Q)$, que enfoca especialmente trastornos psíquicos menores como insomnio, depresión e hipocondriasis? De este instrumento, desarrollado inicialmente por Goldberg en 1972, el propio Goldberg y Williams (1996) ofrecen una Guía actualizada para la aplicación de las distintas versiones del mismo y Badía et al. (1996) lo incluyen en su Guía de escalas de medición en español de las distintas facetas de la salud. Entre los instrumentos trasplantados directamente de la clínica a la psicología del bienestar en el trabajo, destacan asimismo las versiones ampliada y reducida del Beck Depression Inventory, BDI (Beck et al, 1961; Conde y Useros, 1975; Beck, 1978).
Sin embargo, en los últimos lustros, aparecen indicios de un nuevo enfoque que contempla tanto el polo negativo como el positivo de la salud y el bienestar. A ello viene contribuyendo, por un lado, la renovada insistencia por la Organización Mundial de la Salud (1981) en la promoción de la salud como bienestar y no como simple ausencia de malestar, en su estrategia mundial de salud para todos en el año 2000 y en sucesivas propuestas de políticas sanitarias que la han seguido. Y, por otro, el auge de la psicología positiva que, siguiendo el camino iniciado por Marie Jahoda (1958) con su Current Concepts of Positive Mental Health, está contribuyendo a compensar aquel énfasis en lo patológico, reforzando una perspectiva centrada en la evaluación de la vertiente positiva del bienestar personal y laboral (Salanova, Bakker y Llorens, 2006; Salanova y Schaufeli, 2009, Seligman, 2002; Seligman y Csikszentmihalyi, 2000; Snyder y López, 2006; Warr, 2007). Entre los medios disponibles en la actualidad para investigar en esta línea, figuran la Escala de Satisfacción con la Vida (Diener, Emmons, Larsen y Griffin, 1985; Diener, 1994), el Oxford Happiness Questionnaire (Hills y Argyle, 2002), el Quality of Life Enjoyment and Life Satisfaction Questionnaire (Endicott, Nee, Harrison y Blumenthal, 1993), las Escalas de Bienestar Psicológico de Ryff (Ryff y Keyes, 1995; Van Dierendonck, 2004; Díaz et al., 2006) y La Escala de Bienestar Psicológico de Sánchez-Cánovas (1998), que está integrada por cuatro subescalas, una de las cuales referida específicamente al ámbito laboral.

Otro tipo de aportaciones indirectas al estudio del bienestar en el trabajo es el que proviene de la aplicación específica de medidas de bienestar (psicológico, subjetivo) en el marco general de la investigación sobre calidad de vida mediante indicadores sociales, siguiendo una tradición en la que destacan los ya clásicos estudios de Andrews y Crandall (1976), Andrews y Withey (1976) y de Andrews y McKennell (1980) sobre el self reported well-being y escalas como Quality of Well-being Scale (Kaplan y Anderson, 1988) o Happy Life Expectancy (Veenhoven, 1996). En este mismo capítulo pueden incluirse las informaciones obtenidas de la aplicación periódica del World Health Organization Quality Of Life Assessment Instrument, WHOQOL-100- (De Vries y Van Heck, 1997), que incluye, entre otras, medidas específicas de bienestar concernientes a la salud física y psicológica y también a las relaciones sociales. En una línea similar, la New Economics Foundation ha desarrollado el Happy Planet Index (HPI), que incluye aspectos económicos y ecológicos del bienestar humano (Marks et al, 2006; Abdallah et al, 2009). Por su parte, el EuroQol Group viene aplicando un Health Questionnaire para medir "cuán buena o mala es la propia salud hoy" y cuyo resultado final se fija en un punto dentro de una escala que va de 0 (el peor estado de salud imaginable) a 100 (el mejor estado de salud 
imaginable). El común denominador del conjunto de los instrumentos mencionados consiste en que fueron concebidos para la medida del bienestar o la salud (el malestar o la patología) en general; por lo que pueden ser ocasionalmente aplicados al ámbito de la experiencia laboral, como a los de las relaciones familiares o de la vida cotidiana.

En lo que concierne a instrumentos diseñados especialmente para la evaluación de aspectos relacionados de modo directo con el bienestar en el trabajo, uno de los más utilizados es el Maslach Burnout Inventory, en sus diversas versiones y adaptaciones en inglés (Maslach y Jackson, 1981; Maslach, Jackson y Leiter, 1996) o en español (Maslach y Jackson, 1997; GilMonte y Peiró, 1999; Salanova, Schaufeli, Llorens, Peiró y Grau, 2000; Moreno, Rodríguez y Escobar, 2001; Bresó, Salanova, Schaufeli y Nogareda, 2007; etc.). Su versión inicial, el MBI-HSS (Human Services Survey) fue construido para medir fenómenos psicológicamente preocupantes observados en profesionales del servicio a personas, como el trabajo social o la enfermería. Ante determinados problemas prácticos de su aplicación a otro tipo de profesiones, Schaufeli, Leiter, Maslach y Jackson (1996) elaboraron una nueva versión: el MBI-GS (General Survey). A pesar del debate que el modelo teórico del burnout no ha dejado de suscitar desde su primera puesta en escena, las tres dimensiones del mismo en torno a las que se mantiene un mayor consenso son las referidas al agotamiento emocional, a la despersonalización y a la baja realización profesional. El MBI es pues también una escala de medida y evaluación de un síndrome; esto es, para ser aplicada en el marco de la psicopatología del trabajo.

En el contexto de la escasez de herramientas específicas de evaluación del bienestar laboral, ha visto recientemente la luz la Work-Related Quality of Life scale (WRQoWL) (Van Laar et al, 2007; Edwards et al, 2009), que mide 6 factores: satisfacción con el empleo y con la carrera, condiciones de trabajo, bienestar general, conciliación trabajo-hogar, estrés laboral y control en el trabajo. Entre los campos abarcados concretamente por la escala de bienestar general figuran estado de ánimo, depresión, ansiedad, satisfacción vital, calidad de vida general, optimismo y felicidad. Con el ánimo de colaborar en la tarea de ampliar el repertorio de instrumentos de evaluación disponibles en este ámbito, el presente trabajo tiene por objetivo la presentación de la estructura y de las propiedades psicométricas del Cuestionario de Bienestar Laboral General concebido como herramienta específica de evaluación de la dimensiones psicológicas del bienestar en el trabajo, así como del carácter bipolar de esta experiencia. A diferencia de la mayoría de las escalas de bienestar, que suelen referirse a la "vida" en general, con el qBLG se pretende evaluar el bienestar psicológico laboralmente situado.

\section{Método}

\section{Muestra}

De la población de referencia formada por el profesorado y los profesionales de la medicina y de la enfermería que trabajan respectivamente en universidades y en hospitales iberoamericanos, se extrajo una muestra de conveniencia, intencional y estratificada, atendiendo a los criterios de género, generación y profesión. La conformaron 1252 personas, el $48 \%$ de los cuales trabajaba en España, el $18 \%$ en Colombia, el $17 \%$ en Chile, el $14 \%$ en Brasil y el $3 \%$ en Perú. Por sectores profesionales, el $39 \%$ se dedicaba a la docencia e investigación universitarias, el $24 \%$ a la medicina hospitalaria y el $37 \%$ a la enfermería ejercida también en hospitales. Integrada en un $65 \%$ por mujeres y un $35 \%$ por hombres, éstos tuvieron una presencia ligeramente superior en los sectores profesionales de la academia y de la medicina, mientras aquéllas constituyeron la mayoría del de enfermería, como se refleja en la Tabla 1. La edad media de los hombres es de 42.7 años $(\mathrm{SD}=11.3)$ y la de las mujeres de $39.1 \quad(\mathrm{SD}=10.9)$, correspondiendo al sector de enfermería la media de edad más baja, como aparece en la Tabla 2.

Tabla 1. Muestra: Distribución de Sexo por Profesión

\begin{tabular}{lcc}
\hline Profesión & \multicolumn{2}{c}{ Sexo } \\
\hline & Hombres & Mujeres \\
\hline Academia & $53.4 \%$ & $46.6 \%$ \\
Medicina & $55.4 \%$ & $44.6 \%$ \\
Enfermería & $11.3 \%$ & $88.7 \%$ \\
\hline
\end{tabular}

Tabla 2. Muestra: Medias y Desviaciones Típicas de Edades por Profesión

\begin{tabular}{lrr}
\hline Profesión & \multicolumn{2}{c}{ Edad en años } \\
\hline & $\mathrm{M}$ & $\mathrm{SD}$ \\
\hline Academia & 42.30 & 9.98 \\
Medicina & 43.16 & 11.19 \\
Enfermería & 37.33 & 11.47 \\
\hline
\end{tabular}

\section{Instrumento}

En el anexo, se presentan las seis escalas que componen el qBLG, incluyendo las instrucciones de aplicación y el redactado de los ítems. La Figura 1 presenta el modelo teórico que orientó el diseño del cuestionario, según el cual, el bienestar laboral general incluye dos dimensiones principales: bienestar psicosocial (que abarca, a su vez, las facetas de afectos, competencias y expectativas) y efectos colaterales (somatización, desgaste y alienación). Las escalas del factor 
Figura 1. Modelo Teórico de Bienestar Laboral General

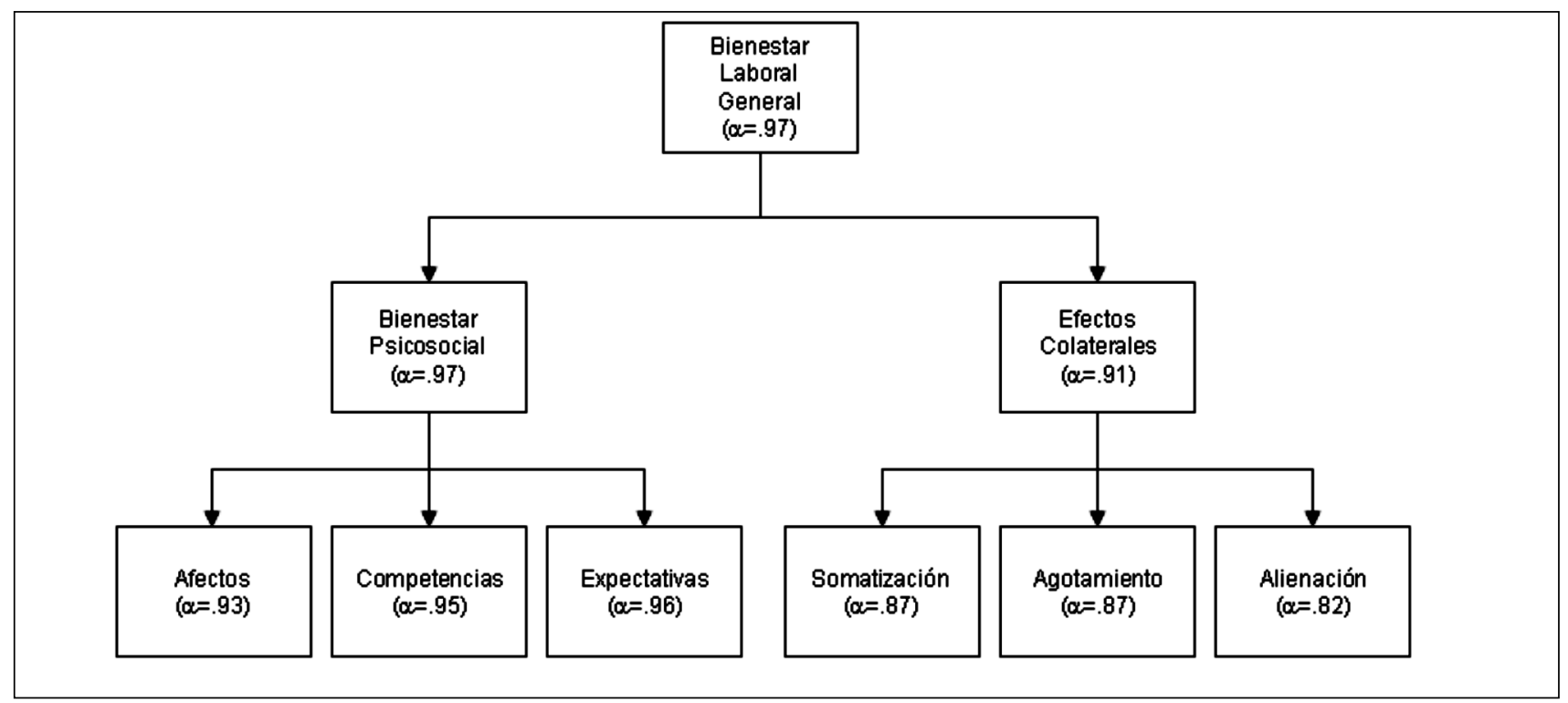

bienestar psicosocial fueron creadas en 2005 para una investigación sobre calidad de vida laboral en el subempleo (Blanch, 2005; Blanch y Cantera, 2008; Goulart, 2006), mostrando buenas propiedades psicométricas (con alfas comprendidas entre 84 y .95). Posteriormente, previa revisión, adaptación y ampliación, fueron aplicadas y revalidadas en otro estudio sobre calidad de vida laboral en universidades y hospitales (Blanch, 2009; Cervantes, 2009). Las del factor somatización fueron especialmente construidas para esta última investigación y, al igual que las demás, puestas a punto en una fase piloto.

Para el diseño de cada una de las dos partes del cuestionario, se procedió a una selección de ítems con criterios relevancia y de representatividad (Camarero, 2001; 2004). Para el cumplimiento del primero de estos criterios, se partió de una revisión de la literatura, que permitió identificar unos tópicos centrales en la investigación sobre el problema. Para el del segundo, se atendió al principio psicométrico de la representatividad teórica (Álvaro, 1997), que remite a la medida en que la "parte" integrada por las dimensiones e indicadores recogidos en el cuestionario representa aspectos importantes de un "todo" tan complejo como el bienestar laboral de profesionales de la docencia universitaria y de la medicina y la enfermería hospitalarias. Para ello, se sometió el borrador del mismo a revisión por una comisión integrada por profesionales de aquellas disciplinas. Los ítems de las dos primeras escalas del factor bienestar psicosocial fueron redactados con formato de diferencial semántico, mientras que para los de las otras cuatro se adoptó un modelo Likert.

Puesto que con el qBLG se pretende evaluar bienestar psicológico laboralmente situado, los encabezamientos de cada escala remiten directa y explícitamen- te a la propia circunstancia laboral. En efecto, para responder las dos primeras, la persona debe ponerse en la siguiente situación: Actualmente, en mi trabajo, siento... Lo mismo acontece con la tercera: En mi trayectoria laboral... Y con las tres restantes: Actualmente, por causa de mi trabajo, siento...

\section{Procedimiento}

El qBLG, de autoaplicación individual a base de lápiz y papel, estuvo incluido en uno más amplio, que integraba también, entre otras, escalas de condiciones de trabajo, clima social, satisfacción con la organización y cultura organizacional. El tiempo medio de la aplicación de este instrumento global osciló en torno a los 30 minutos; a los que se añadieron, en la mayoría de los casos, otros 25 correspondientes a una entrevista semiestructurada y, en algunos otros, la participación en algún grupo focal.

Considerada la tipología del personal a investigar, supuestamente con poco tiempo disponible, no se estimó oportuno acceder al mismo por azar (por el previsible bajo porcentaje de respuesta). Tampoco se hizo de arriba hacia abajo, buscando la complicidad de la dirección de las organizaciones (universitarias y hospitalarias), para garantizar las máximas cotas posibles de credibilidad, confidencialidad y anonimato. Así que se hizo de modo individualizado en cada caso, por la vía de las redes de contactos personales de los miembros de los equipos de cada país. Una vez una persona contactada accedía a responder el cuestionario, que le era presentado como parte de un estudio internacional sobre calidad de vida laboral en universidades y hospitales, se la invitaba a firmar una hoja de consentimiento informado. Tanto en esta hoja 
Figura 2. Gráfico de Sedimentación de Componentes Principales de las Escalas de Bienestar Psicosocial

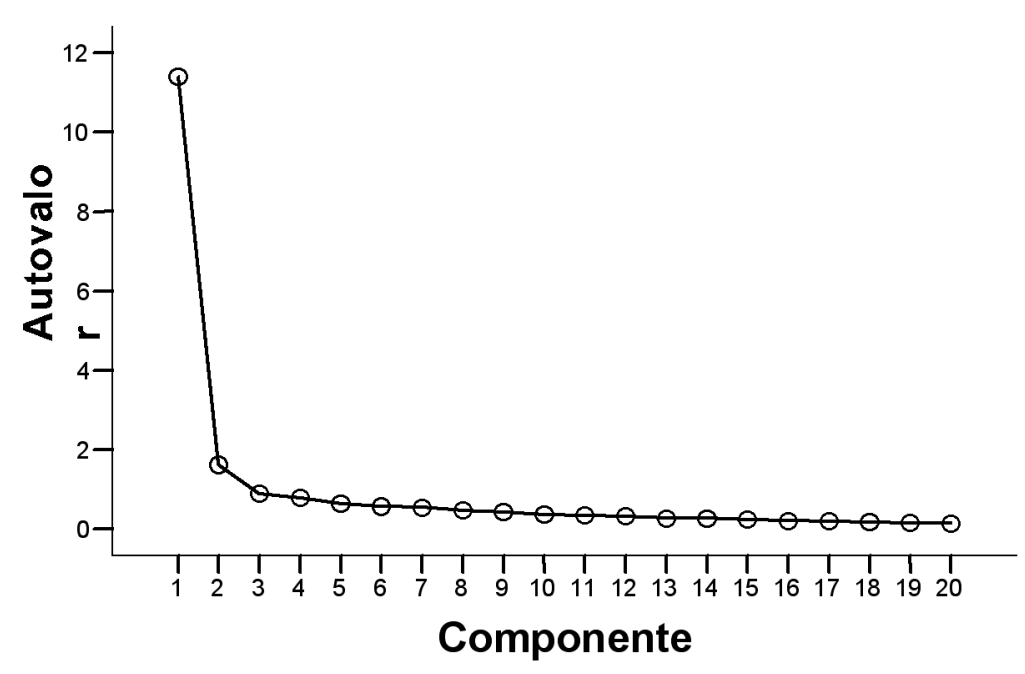

como en la portada del cuestionario, aparecía el nombre, la dirección y los datos para la localización electrónica y telefónica de los responsables general y local de la investigación. La información de cada cuestionario fue introducida on line por cada uno de los grupos de investigación participantes en el proyecto a la base de datos común incluida en la Web de la red de trabajo.

La valoración psicométrica del cuestionario se realizó en dos fases. En una primera fase, se valoró la validez de contenido del instrumento por medio de una revisión de literatura y de un consejo de jueces. En la fase siguiente, se abordó en primer lugar lo relativo a validez de constructo mediante un análisis de componentes principales de los ítems incluidos en cada uno de los dos grupos de escalas (escalas de bienestar psicosocial y escalas de efectos colaterales), siguiendo las recomendaciones de Clark y Watson (1995). Este análisis se complementó con otro análisis de componentes principales del conjunto de puntajes (sumatorias de ítems) de cada una se las seis escalas. Posteriormente, se realizó un análisis de fiabilidad, calculando el coeficiente Alfa de Cronbach (1951) para cada una de las seis escalas incluidas en el cuestionario (Afectos, Competencias, Expectativas, Somatización, Desgaste y Alienación), para el conjunto de ítems incluidos en cada uno de los dos factores generales (bienestar psicosocial y efectos colaterales) y para la totalidad de ítems incluidos en el cuestionario.

\section{Resultados}

El análisis componentes principales con rotación promax para las series de ítems integrados en cada uno de los dos grupos de escalas (bienestar psicosocial y efectos colaterales) confirmó la existencia de tres com- ponentes distintos dentro de cada grupo y la correspondencia de cada componente con cada una de las tres escalas, tal como proponía el modelo teórico.

En la Figura 2, se presenta el gráfico de sedimentación de las escalas de bienestar psicosocial, mostrando la existencia de tres grandes componentes. Por su parte, la Tabla 3 muestra la estructura factorial de las escalas obtenida usando rotación promax, así como los autovalores iniciales y la varianza explicada (64\% para los tres primeros componentes).

Con resultados semejantes, la Figura 3 presenta el gráfico de sedimentación resultante del análisis de componentes principales de las escalas incluidas en el segundo grupo (efectos colaterales). Siguiendo el mismo procedimiento de rotación (promax), la Tabla 4 muestra la estructura factorial de las escalas de este grupo, los autovalores iniciales y la varianza explicada (69\% para los tres primeros componentes).

El primer grupo de escalas (bienestar psicosocial) está compuesto por 42 ítems, agrupados a su vez en 3 componentes o factores específicos: las escalas de Afectos (10 ítems), de Competencias (10 ítems) y de Expectativas (22 ítems). Por otra parte, componen el segundo grupo de escalas (efectos colaterales) 3 componentes o factores específicos: las escalas de Somatización (5 ítems), de Desgaste (4 ítems) y de Alienación (4 ítems).

El análisis de componentes principales (rotación varimax) del conjunto de puntajes ${ }^{1}$ (sumatoria de ítems) de las seis escalas del qBLG, puso al descubierto dos componentes o factores generales con autovalores superiores a 1, que coinciden con las dos dimensiones del bienestar laboral general propuestas por el modelo teórico que orientó la investigación y que

\footnotetext{
${ }^{1}$ En todos los casos, los puntajes de cada escala fueron calculados sumando los valores de los ítems incluidos en la escala.
} 
Tabla 3. Estructura Factorial de las Escalas de Bienestar Psicosocial (Rotación Promax)

\begin{tabular}{|c|c|c|c|}
\hline & \multicolumn{3}{|c|}{ Componentes } \\
\hline & 1 & 2 & 3 \\
\hline Mi motivación por el trabajo & .798 & .322 & .559 \\
\hline Mi identificación con los valores de la org. & .749 & .402 & 697 \\
\hline Mi rendimiento profesional & .793 & .389 & .398 \\
\hline Mi capacidad de gestión de mi carga de trabajo & .710 & .305 & .351 \\
\hline La calidad de mis condiciones de trabajo & .676 & .327 & .707 \\
\hline Mi autoestima profesional & .813 & .388 & .591 \\
\hline La cordialidad en mi ambiente social de trabajo & .650 & .421 & .602 \\
\hline La conciliación de mi trabajo con mi vida privada & .576 & .303 & .555 \\
\hline Mi confianza en mi futuro profesional & .709 & .364 & .709 \\
\hline Mi calidad de vida laboral & .735 & .389 & .766 \\
\hline El sentido de mi trabajo & .835 & .430 & .608 \\
\hline Mi acatamiento de las pautas de la dirección & .642 & .340 & .550 \\
\hline Mi estado de ánimo laboral & .811 & .415 & .726 \\
\hline Mis oportunidades de promoción laboral & .658 & .339 & .697 \\
\hline Mi sensación de seguridad en el trabajo & .584 & .413 & .788 \\
\hline Mi participación en las decisiones de la org. & .564 & .340 & .696 \\
\hline Mi satisfacción con el trabajo & .817 & .415 & .711 \\
\hline Mi realización profesional & .811 & .409 & .662 \\
\hline El nivel de excelencia de mi org. & 639 & .368 & 649 \\
\hline Mi eficacia profesional & .804 & .422 & .472 \\
\hline Mi compromiso con el trabajo & .803 & .394 & .432 \\
\hline Mis competencias profesionales & .766 & .394 & .425 \\
\hline Insensibilidad - Sensibilidad & .411 & .811 & .505 \\
\hline Irracionalidad - Racionalidad & .392 & .828 & .447 \\
\hline Incompetencia - Competencia & .396 & .841 & .463 \\
\hline Inmoralidad - Moralidad & .347 & .838 & .439 \\
\hline Maldad - Bondad & .320 & .813 & .439 \\
\hline Fracaso - Éxito & .451 & .797 & .661 \\
\hline Incapacidad - Capacidad & .422 & .856 & .587 \\
\hline Pesimismo - Optimismo & .529 & .814 & .714 \\
\hline Ineficacia - Eficacia & .425 & .840 & .523 \\
\hline Inutilidad - Utilidad & .392 & .841 & .474 \\
\hline Insatisfacción - Satisfacción & .636 & .547 & .745 \\
\hline Inseguridad - Seguridad & .365 & .512 & .725 \\
\hline Intranquilidad - Tranquilidad & .405 & .578 & .776 \\
\hline Impotencia - Potencia & .484 & .633 & .761 \\
\hline Malestar - Bienestar & .521 & .717 & .754 \\
\hline Desconfianza - Confianza & .463 & .732 & .748 \\
\hline Incertidumbre - Certidumbre & .363 & .629 & .731 \\
\hline Confusión - Claridad & .455 & .776 & 683 \\
\hline Desesperanza - Esperanza & .453 & .778 & 671 \\
\hline Dificultad - Facilidad & .408 & .600 & .707 \\
\hline Autovalores iniciales & 20.60 & 4.46 & 1.82 \\
\hline$\%$ de Varianza & 49.05 & 10.56 & 4.34 \\
\hline$\%$ Acumulado de Varianza & 49.05 & 59.61 & 63.94 \\
\hline
\end{tabular}

explican, en conjunto, el $77 \%$ de la varianza total. La Tabla 5 muestra esta estructura factorial.

En cuanto al análisis de fiabilidad del cuestionario y los factores y escalas que lo componen, la Figura 1 presenta el modelo general en el que se fundamentan, incluyendo las puntuaciones alfa de Cronbach obtenidas para el conjunto del cuestionario, así como para cada uno de sus factores y escalas. Los valores alfa oscilan entre 0.82 , correspondiente a la escala con menor número de ítems (4) y 0.96 , correspondiente la escala con mayor número de los mismos (22).

\section{Discusión}

Los resultados obtenidos indican que la estructura del qBLG reproduce fielmente el modelo teórico utilizado como base para esta investigación (Figura 1) y que este cuestionario constituye un instrumento psicométricamente adecuado para la evaluación del bienestar laboral general de personal que trabaja en organizaciones de servicio a personas, como en el caso de profesionales de la academia y de la sanidad empleados respectivamente en universidades y en hospitales. El instrumento, que 
Figura 3. Gráfico de Sedimentación de Componentes Principales de las Escalas de Efectos Colaterales

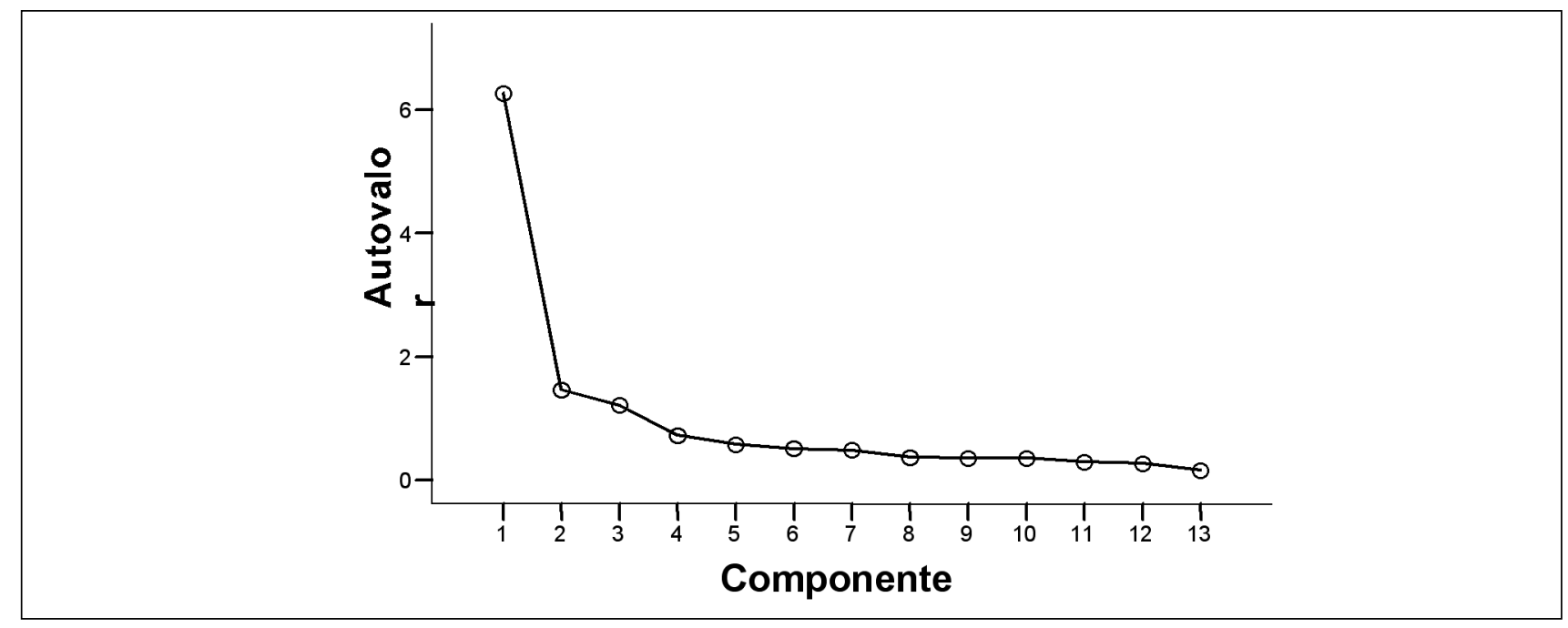

Tabla 4. Estructura Factorial de las Escalas de Efectos Colaterales*

\begin{tabular}{lrr}
\hline & \multicolumn{2}{c}{ Componentes } \\
\cline { 2 - 3 } & \multicolumn{1}{c}{1} & \multicolumn{1}{c}{2} \\
\hline Afectos $^{* *}$ & $\mathbf{. 9 1 3}$ & -.221 \\
Competencias $^{* *}$ & $\mathbf{. 9 0 1}$ & -.050 \\
Expectativas $^{* *}$ & $\mathbf{. 9 3}$ & -.288 \\
Somatización $^{* *}$ & -.106 & $\mathbf{. 8 6 1}$ \\
Desgaste $^{* *}$ & -.137 & $\mathbf{. 8 6 8}$ \\
Alienación $^{* *}$ & -.494 & $\mathbf{. 6 7 8}$ \\
\hline Autovalores iniciales $_{\% \text { de Varianza }}$ & 3.36 & 1.27 \\
\% Acumulado de Varianza & 56.09 & 21.21 \\
\hline${ }^{*}$ Rotación Varimax & 56.09 & 77.30 \\
${ }^{* *}$ Puntajes (sumatorias) de escalas & & \\
\hline
\end{tabular}

incluye dos escalas con formato de diferencial semántico y cuatro de tipo Likert, reúne condiciones satisfactorias en cuanto a fiabilidad y validez. La consistencia interna de las escalas es alta y el proceso de su construcción se adecuó a los estándares metodológicos.

El qBLG tiene además algunas características especiales: por una parte, permite evaluar un bienestar laboralmente situado; es decir, sensible a las particularidades de este ámbito específico. Por otra, trata el bienestar en el trabajo como un todo integrado; posibilitando un abordaje de las caras positiva y negativa de esta experiencia. Además, ha sido producido en el contexto iberoamericano y aplicado en el ámbito específico de actividades profesionales de servicio a personas. Más allá de su utilidad como herramienta de investigación, el qBLG ha sido desarrollado de tal forma que en un futuro se puedan concretar condiciones y pautas para su uso práctico como herramienta de diagnóstico. Ello comportaría su tipificación en función de los dis-
Tabla 5. Estructura Factorial del Cuestionario de Bienestar Laboral General*

\begin{tabular}{lccc}
\hline & \multicolumn{3}{c}{ Componentes } \\
\cline { 2 - 4 } & 1 & 2 & 3 \\
\hline Trastornos digestivos & $\mathbf{. 7 4}$ & .420 & .483 \\
Dolores de cabeza & $\mathbf{. 8 2 6}$ & .514 & .405 \\
Insomnio & $\mathbf{. 7 4 5}$ & .435 & .463 \\
Dolores de espalda & $\mathbf{. 8 4 6}$ & .508 & .323 \\
Tensiones musculares & $\mathbf{. 8 6 7}$ & .541 & .345 \\
Sobrecarga de trabajo & .412 &. $\mathbf{7 9 2}$ & .257 \\
Desgaste emocional & .526 & $\mathbf{. 8 4 4}$ & .564 \\
Agotamiento físico & .550 &. $\mathbf{8 6 6}$ & .420 \\
Saturación mental & .545 & $\mathbf{. 8 7 7}$ & .521 \\
Mal humor & .505 & .657 & $\mathbf{. 7 4 6}$ \\
Baja realización profesional & .392 & .432 & $\mathbf{. 8 4 0}$ \\
Trato despersonalizado & .286 & .281 &. $\mathbf{7 5 8}$ \\
Frustración & .460 & .441 & $\mathbf{. 8 5 0}$ \\
\hline Autovalores iniciales & 6.28 & 1.42 & 1.21 \\
\% de Varianza & 48.30 & 10.95 & 9.34 \\
\% Acumulado de Varianza & 48.30 & 59.26 & 68.61 \\
\hline
\end{tabular}

"Rotación Promax

tintos ámbitos profesionales en los que podría ser utilizado y, paralelamente, la investigación de las relaciones entre sus factores y escalas y los de otros instrumentos de evaluación.

En esta línea, se estudiaron las correlaciones entre las escalas del factor bienestar psicosocial y la escala de satisfacción con la organización ${ }^{2}$, que también formó parte de la encuesta en la que estuvo integrado qBLG. En este análisis, los puntajes de las escalas de 2009). 
Bienestar Psicosocial mostraron correlaciones altas y significativas $(p<.001)$ con el de la escala de Satisfacción con la Organización: $r=.65$ en el caso de la escala de Afectos; $r=.50$ en la escala de Competencias y $r=.75$ en la de Expectativas.

Asimismo, en un estudio paralelo en curso, con una muestra de características demográficas homogéneas con respecto a las de la que respondió el qBLG, los participantes (182 sujetos hasta el momento) respondieron el qBLG conjuntamente con la versión española del Maslach Burnout Inventory elaborada por Bresó et al. (2007). En la comparación de las puntuaciones entre las diversas escalas, se observó que las del factor general Efectos Colaterales correlacionaron positiva- mente con las escalas de Desgaste Emocional y de Despersonalización o Cinismo del MBI, como en el caso de la de Desgaste del qBLG con el factor Desgaste Emocional del MBI $(r=.67, p<.001)$. Algo semejante ocurrió con las escalas del factor general de Bienestar Psicosocial, que mostraron correlaciones positivas con el factor Eficacia Profesional del MBI, como en el caso de la escala Competencias del qBLG $(r=.42, p<.001)$. Con tales estudios se pretende consolidar progresivamente la utilidad de un instrumento fundamentado teórica y empíricamente para abordar múltiples facetas de un fenómeno tan complejo como la experiencia de bienestar en un ámbito no menos complejo como el entorno de trabajo.

\section{Extended Summary}

Since the time when the World Health Organization defined health as "a state of complete physical, psychological and social well-being", the complex and intense relationship between well-being and work have focused the interest of psychology in general and particularly of the work and organizational psychology. According to its most commonly accepted meaning, well-being (subjective or psychological) is a set of value judgments and emotional reactions concerning the extent to which the life is experienced as satisfactory, pleasant and positive (Andrews \& Withey, 1976; Diener, 2000; Diener \& Suh, 2001; Diener, Oishi \& Lucas 200;, Lucas, Diener \& Suh, 1996).

In the experience of "being well" in life and at work, environmental and personal factors converge. The dialectical game between "situation centered" and "person centered" explanations of well-being is marked by the predominance of the clinical point of view, which in turn reinforces the pathologist bias of the psychology of well-being at work. According to Schaufeli and Backer (2004), until the early twentyfirst century, psychological research on negative aspects of work experience (distress, burnout, anxiety, depression, physical and mental disorders, etc.) prevailed with a ratio of 15 to 1 upon the study on its positive aspects (happiness, satisfaction, well being, self, etc.).

One of the most frequently used tools for assessing employee well-being in terms of discomfort is the General Health Questionnaire (GHQ, Goldberg, 1972; Goldberg \& Williams, 1996), which focuses especially minor psychiatric disorders such as insomnia, depression and hypochondriasis. Another one is the Beck Depression Inventory (BDI, Beck et al, 1961; Conde Useros, 1975; Beck, 1978). Among the instruments specially designed for the evaluation of issues directly related to psychopathology in the workplace, highlights the Maslach Burnout Inventory, in its various versions and adaptations in English (Maslach \&
Jackson, 1981; Maslach, Jackson \& Leiter, 1996) or Spanish (Bresó, Salanova, Schaufeli \& Nogareda, 2007; Maslach \& Jackson, 1997; Gil-Monte \& Peiró, 1999; Salanova, Schaufeli, Llorens, Peiró \& Grau, 2000; Moreno, Rodríguez \& Escobar, 2001, etc.) The three main dimensions of the burnout theoretical model are those affecting to the emotional exhaustion, to depersonalization or cynicism, and to lack of professional realization (efficacy).

However, in recent decades, there are signs of a new look that includes both the negative and positive health and well-being approaches. The renewed insistence of World Health Organization on the positive side of health, and the emergence of Positive Psychology, following the path initiated by Marie Jahoda (1958) with its Current Concepts of Positive Mental Health, push to make emphasis on the positive side of working experience and of personal wellbeing (Salanova, Bakker \& Llorens, 2006; Salanova \& Schaufeli, 2009; Seligman, 2002; Seligman \& Csikszentmihalyi, 2000; Snyder \& Lopez, 2006; Warr, 2007). The available means to investigate on this line include the Life Satisfaction Scale (Diener, Emmons, Larsen \& Griffin, 1985; Diener, 1994), the Oxford Happiness Questionnaire (Hills and Argyle, 2002), the Quality of Life Enjoyment and Life Satisfaction Questionnaire (Endicott, Nee, Harrison, \& Blumenthal, 1993), the Ryff Psychological Well-Being scales (Díaz et al., 2006; Ryff \& Keyes, 1995; Van Dierendonck, 2004), and Sanchez-Cánovas (1998) Psychological Well Being Scale, one of which four subscales referred specifically to the workplace experience.

Another type of indirect contributions to the study of well-being at work is coming from the specific implementation of well-being measures (psychological, subjective) as part of the research on quality of life through social indicators, following a tradition that include the now classic studies by Andrews and Crandall (1976), Andrews and Withey (1976) and 
Andrews and McKennell (1980) on The Self Reported Well-Being, and the Quality of Well-being Scale (Kaplan \& Anderson, 1988), the Happy Life Expectancy Scale (Veenhoven, 1996), and the World Health Organization Quality Of Life Assessment Instrument, WHOQOL-100-(De Vries \& Van Heck, 1997), which includes, among others, specific measures of well-being concerning the physical and psychological health and social relations.

Concerning the specific tools of assessment of work well-being, the Work-Related Quality of Life scale (WRQoWL) (Van Laar et al, 2007; Edwards et al, 2009) measures 6 factors: job satisfaction and career, working conditions, well-being, work-home balance, work stress and job control. Among the areas specifically covered by the general well-being scale set mood, depression, anxiety, life satisfaction, overall quality of life, optimism and happiness.

The common denominator of most of these instruments is that they are designed for the measurement of well-being or health (discomfort or pathology) in general, hence it may occasionally be applied to the field of work experience, as the family relationships or everyday life. With the aim of collaborating in the task of expanding the repertoire of assessment tools available in this field, this paper aims at presenting the structure and the psychometric properties of a General Work Well-being Questionnaire (qBLG) designed as a tool for specific assessment of the psychosocial dimension of well-being at work, and of the bipolar nature of this experience. Unlike most well-being scales often refer to "life" in general, the object of the qBLG is to assess the work situated psychological well-being.

\section{Method}

\section{Sample}

From a reference population made up of teachers and medical professionals and nurses working respectively in Latin American universities and hospitals, we pulled a convenience sample, intentional and stratified according to criteria of gender, generation and profession. The sample was composed by 1252 people, $48 \%$ of whom worked in Spain, $18 \%$ in Colombia, $17 \%$ in Chile, $14 \%$ in Brazil and $3 \%$ in Peru. For professional sectors, 39\% were academic workers, and $24 \%$ medical and $37 \%$ nursing employed in hospitals. $65 \%$ of the sample was women and $35 \%$ men, who had a slightly higher presence in professional fields of academia and medicine, while women made up most of nursing, as reflected in Table 1. The average age for men was 42.7 years $(\mathrm{SD}=11.3)$ and for women $39.1(\mathrm{SD}=10.9)$, corresponding to the nursing sector the average age lower, as shown in Table 2 .

\section{Instrument}

The annex presents the six scales of the qBLG, including application instructions and the wording of the items. Figure 1 represents the theoretical model that guided the design of the questionnaire, according to which general welfare work includes two main dimensions: psychosocial well-being (covering, in turn, the facets of affect, skills and expectations) and side effects (somatization, wear and alienation).

The scales of psychosocial well-being factor were created in 2005 for research on quality of working life in the underemployment (Blanch, 2005, 2006, Goulart, 2006, Blanch \& Cantera, 2008), showing good psychometric properties (with alphas ranging from .84 and 95). Later, after review, adaptation and enlargement, were applied and re-validated in another study on quality of working life in universities and hospitals (Blanch, 2009; Cervantes, 2009). The somatization factor was specially built for this latest research and, like others, made a point in a pilot phase. The items of the first two scales of the psychosocial well-being factor were drafted with semantic differential format, while for the other four Likert model was adopted. Since the qBLG is to assess psychological well-being occupationally situated, the headings of each scale directly and explicitly refer to the own work situation.

\section{Procedure}

The qBLG, with pencil and paper format, was individually self-administered by people who were accessed via networks of personal contacts of members of the teams in each country. Once a person contacted agreed to answer the questionnaire, which was submitted as part of an international study of quality of working life in universities and hospitals, was requested to sign an informed consent form.

\section{Results}

Principal components analysis with promax rotation for integrated series of items in each of the two sets of scales (psychosocial well-being and side effects) confirmed the existence of three distinct components within each group and the correspondence of each component with each of the three scales, as suggested by the theoretical model.

Figures 2 and 3 present the graph of sedimentation of the scale, indicating the existence of three major components in each one of them. For its part, Tables 3 and 4 show the factor structure of the scales obtained using promax rotation, and their initial eigenvalues and the percentage of variance explained.

The principal component analysis (varimax rotation) of all scores (sum of items) of the six scales of 
qBLG, revealed two components or general factors with eigenvalues greater than 1 , which coincide with the two dimensions of general work well-being proposed by the theoretical model that guided the investigation. They explain, as a whole, $77 \%$ of the total variance. Table 5 shows the factor structure.

As for the reliability analysis, Figure 1 presents the Cronbach alpha scores obtained for the entire questionnaire and for each of its factors and scales. The alpha values range from 0.82 , corresponding to the scale with fewer items (4) and 0.96 , corresponding to the scale with the largest number of them (22).

\section{Discussion}

The results indicate that the structure of qBLG faithfully reproduces the theoretical model used as the basis for this investigation (Figure 1) and that this questionnaire is a psychometrically adequate instrument for the assessment of general work well-being of personnel employed in service organizations as professionals from academia and health care. The instrument, which includes two scales with semantic differential format and four with Likert format, meet satisfactory conditions in terms of reliability and validity. The internal consistency of the scales is high and the process of its construction was adapted to the methodological standards.

The qBLG also has some special features. Firstly, it allows evaluating work situated well-being (sensitive to the particularities of this specific field). In addition, it evaluates work well-being as an integrated whole, looking at positive and negative sides of this experience. This questionnaire has been produced in the IberoAmerican context and applied in the specific field of professional service to people.

Concerning relationships among its elements and scales and other assessment tools, we studied the correlations between the scales of psychosocial wellbeing factor and the level of satisfaction with the organization, which also took part in the survey that consisted qBLG. In this analysis, the scores for psychosocial well-being scales showed high and significant correlations $(p<.001)$ with the level of satisfaction with the organization: $\mathrm{r}=0.65$ in the case of Affective scale, $r=.50$ in Competence scale, and $r=$ 0.75 in Expectations scale. Also, in a parallel study under way, with a sample of homogeneous demographic characteristics with respect to those that answered the qBLG, participants (182 subjects to date) responded along with the qBLG a Spanish version of the Maslach Burnout Inventory developed by Bresó et al. (2007). In the comparison of scores between different scales, it was observed that the general factor Side Effects correlated positively with the scales of emotional exhaustion and depersonalization or cynicism of the MBI, as in the case of the Wear factor of qBLG with Emotional Wear MBI $(\mathrm{r}=0.67, p<.001)$.
Something similar happened with the scales of Psychological Well-Being factor, which showed positive correlations with the MBI Professional Efficacy factor, as in the case of the Skills of the qBLG scale $(\mathrm{r}=.42, p<.001)$.

Such studies may contribute to gradual consolidation of the utility of a theoretically and empirically based instrument to address multiple facets of a phenomenon as complex as the experience of well-being in an area not less complex as the work environment.

\section{Referencias}

Abdallah, S., Thompson, S., Michaelson, J., Marks, N., Steuer, N. et al. (2009). The Happy Planet Index 2.0. London: New Economics Foundation.

Alvaro, M. (1997). Principios psicométricos de la evaluación psicológica. In G. Buela y J.C. Sierra, (Coords.). Manual de Evaluación Psicológica. Fundamentos, técnicas y aplicaciones. (pp. 173-192). Madrid: Siglo XXI.

Andrews, F.M. y McKennell, A.C. (1980). Measures of self reported well-being: Their affective, cognitive and other components. Social Indicators Research, 8, 127-155.

Andrews, F.M. y Withey, S.B. (1976). Social Indicators of Well-Being: Americans Perceptions of Life Quality. Nueva York: Plenum Press.

Andrews, F.M. y Crandall, R. (1976). The validity of measures of self-reported well-being. Social Indicators Research, 3, 1-19.

Avia, M. D. y Vázquez, C. (1998). Optimismo Inteligente. Madrid: Alianza.

Badía, X., Salamero, M., Alonso, J. y Ollé, A. (1996). La medida de la salud. Guía de escalas de medición en español. Barcelona: PPU.

Beck, A.T. (1978). Inventario de Depresión. Revisión. In A.T. Beck, et al. Terapia Cognitiva de la Depresión (pp.358-360). Bilbao: DDB.

Beck, A.T., Ward, C.H., Mendelson, M., Mock, J. y Erbaugh, J. (1961). An inventory formeasuring depression. Archives of general psychiatry, 4, 461-571.

Blanch, J.M. (2006). Estudio internacional sobre la calidad de la experiencia laboral $e$ el capitalismo flexible. In X. Díaz, L. Godoy, A. Stecher y J. P. Toro (Coords.). Trabajo, identidad y Vínculo Social. Reflexiones y experiencias en el capitalismo flexible (pp. 75-107). Santiago de Chile: CEM- UDP.

Blanch, J.M. (2005). Estudio de calidad de vida laboral en el subempleo. In J. Romay, J. Salgado, M. Romaní y D. Robla (Comps.). Psicología de las Organizaciones, del Trabajo y Recursos Humanos y de la Salud (pp.79-87). Madrid: Biblioteca Nueva.

Blanch, J.M. (2009). Propiedades psicométricas de las escalas del cuestionario. Estudio piloto. Documento interno de trabajo del grupo investigación Colaborando. Universidad Autónoma de Barcelona.

Blanch, J.M. y Cantera, L. (2009). El Malestar en el Empleo Temporal Involuntario. Malaise in Involuntary Tempora- 
ry Employment. Revista de Psicología del Trabajo y las Organizaciones. Journal of Work and Organizational Psychology. 25, 59-70.

Blanco, A., y Díaz, D. (2005). El bienestar social: su concepto y medición. Psicothema, 17 (3), 580-587.

Bresó, E., Salanova, M., Schaufeli, W. B. y Nogareda, C. (2007). Síndrome de estar quemado por el trabajo "Burnout" (III): Instrumento de medición. Nota Técnica de Prevención, 732, $21^{\text {a }}$ Serie. Madrid: Instituto Nacional de Seguridad e Higiene en el Trabajo.

Camarero L. (2001). Los soportes de la encuesta. La infancia de los métodos representativos. Metodología de Encuestas, 3, 163-181.

Camarero, L. (2004). Representatividad estadística versus social. Metodología de Encuestas, 6, 61-70.

Cervantes, G (2009). Calidad de vida laboral y riesgos psicosociales en profesionales de la salud catalanes. De la presión asistencial a la violencia ocupacional. Tesis doctoral no publicada. Departamento de Psicología Social. Universidad Autónoma de Barcelona.

Clark, L. A. y Watson, D. (2003). Constructing validity: Basic issues in objective scale development. Psychological Assessment, 7, 207-231.

Conde, V. y Useros, E. (1975). Adaptación castellana de la escala de evaluación conductual para la depresión de Beck. Revista de psiquiatría y psicología médica, 12 (4), 217-236.

Csikszentmihalyi, M. (1990). Flow. The Psychology of Optimal Experience. Harper Collins.

De Cuyper, N., Isaksson, K. y De Witte, H. (Eds). (2005). Employment contracts and well-being among European workers. Aldershot: Ashgate.

De Vries, J. y Van Heck, G.L. (1997). The World Health Organization Quality od Life Assessment Instrument (WHOQOL-100): Validation study with the Dutch Version. European journal of psychological assessment, 13, 164-178.

De Witte, H. (1999). Job insecurity and psychological wellbeing. Review of the literature and exploration of some unresolved issues. European Journal of Work and Organizational Psychology, 8, 155-177.

Díaz, D., Rodríguez-Carvajal, R., Blanco, A., MorenoJiménez, B., Gallardo, I, Valle, C. y van Dierendonck, D. (2006) Adaptación española de las escalas de bienestar psicológico de Ryff. Psicothema. 18, 572-577.

Diener, E. (1984). Subjective Well-Being. Psychological Bulletin, 95 (3), 542-575. (Trad: El bienestar subjetivo. Intervención Psicosocial, 8, 67-113).

Diener, E. (1994). Assessing Subjective Well-Being: Progress and Opportunities. Social Indicators Research, 31, 103-157.

Diener, E. (2000). Subjective well-being: The science of happiness and a proposal for a national index. American Psychologist, 55, 34-43.

Diener, E.D., Emmons, R.A., Larsen, R.J. y Griffin, G. (1985). The Satisfaction With Life Scale. Journal of Personality Assessment, 49, 71-75.

Diener, E., Oishi, S. y Lucas, R. E. (2003). Personality, culture, and subjective well-being: Emotional and cognitive evaluations of life. Annual Review of Psychology, 54, 403-425.

Diener, E. y Suh, E. (2001). Culture and Subjective WellBeing. Cambridge, Ma: MIT Press.

Edwards, J., Van Laar, D.L. y Easton, S. (2009). The WorkRelated Quality of Life (WRQoL) scale for Higher Education Employees. Quality in Higher Education, 15, 207-219.

Endicott, J., Nee, J., Harrison, W. y Blumenthal, R. (1993). Quality of Life Enjoyment and Life Satisfaction Questionnaire: a new measure. Psychopharmacological Bulletin, 28, 321-326.

Gil-Monte, P. R. y Peiró, J. M. (1999) Validez factorial del Maslach Burnout Inventory en una muestra multiocupacional. Psicothema, 11, 679-689.

Goldberg, D. y Williams, P. (1996). Cuestionario de salud general GHQ (General Health Questionnaire). Guía para el usuario de las distintas versiones. Barcelona: Masson.

Goulart, P M (2006). Trabajar en el nuevo capitalismo. Cultura y experiencia del trabajo en el Brasil contemporáneo. Tesis doctoral no publicada. Departamento de Psicología Social, Universidad Autónoma de Barcelona.

Hills, P., y Argyle, M. (2002). The Oxford Happiness Questionnaire: a compact scale for the measurement of psychological well-being. Personality and Individual Differences, 33, 1073-1082.

Jahoda, M. (1958). Current concepts of positive mental health. New York: Basic Books.

Kaplan, R. M. y Anderson J. P. (1988). The Quality of Wellbeing Scale: rationale for a single Quality of life index. En S. R. Walker y R.M. Rosser (Eds.) Quality of Life: assessment and applications (pp. 51-77). Lancaster: MTP.

Keyes, C., Ryff, C. y Shmotkin, D. (2002). Optimizing wellbeing: the empirical encounter of two traditions. Journal of Personality and Social Psychology, 82, 1007-1022.

Lucas, R. E. y Diener, E. (2003).The happy worker: Hipothesis about the role of positive affect in worker productivity. In M. R. Barrick y A. M. Ryan (Eds.). Personality and Work (pp. 30-59). San Francisco: Jossey-Bass.

Lucas, R. E., Diener, E. y Suh, E. (1996). Discriminant validity of well-being measures. Journal of Personality and Social Psychology, 71, 616-628.

Marks, N., Abdallah, S., Simms, A. y Thompson, S. (2006). The Happy Planet Index 1.0. London: New Economics Foundation.

Maslach, C. y Jackson, S. E. (1981). Maslach Burnout Inventory. Palo Alto: CA: Consulting Psychologists Press. (Versión española en Maslach, C. y Jackson, S. E. (1997). MBI. Inventario Burnout de Maslach. Síndrome del Quemado por estrés asistencial. Madrid: TEA.)

Maslach, C., Jackson, S. E. y Leiter, M.P. (1996). Maslach Burnout Inventory Manual $\left(3^{\text {rd }}\right.$. Ed). Palo Alto: CA: Consulting Psychologists Press.

Moreno, B; Rodríguez, R. y Escobar, E. (2001). La evaluación del burnout profesional. Factorialización del MBIGS. Ansiedad y Estrés, 7, 69-78 
Organización Mundial de la Salud (OMS) (1981). Estrategia mundial de salud para todos en el año 2000. Ginebra: Organización Mundial de la Salud, Serie "Salud para todos", no 3 .

Peralta, M C (2010). Entre la formalidad y la informalidad. Construcción de sujetos laborales en el nuevo contexto colombiano. Tesis doctoral. Programa Doctorado Psicología Social. Universidad Autónoma de Barcelona. (JM Blanch Dir.)

Ryff, C., y Keyes, C. (1995). The structure of psychological well-being revisited. Journal of Personality and Social Psychology, 69, 719-727.

Salanova, M., Bakker, A. y Llorens, S. (2006). Flow at Work: Evidence for a Gain Spiral of Personal and organizational Resources. Journal of Happiness Studies, 7, 122.

Salanova, M., Schaufeli, W. B., Llorens, S., Peiró, J. M., y Grau, R. (2000). Desde el "burnout" al "engagement": ¿Una nueva perspectiva? Revista de Psicología del Trabajo y de las Organizaciones, 16, 117-134.

Sánchez-Cánovas, J. (1998). Escala de bienestar psicológico. Madrid: TEA.

Schaufeli, W.B., Leiter, M.P., Maslach, C. y Jackson, S.E. (1996). Maslach Burnout Inventory - General Survey. In C.Maslach, S.E. Jackson, \& M. P. Leiter: The Maslach Burnout Inventory-Test Manual (3rd ed.). Palo Alto, CA: Consulting Psychologists Press.

Seligman, M. E. P. (2002). Authentic Happiness: Using the New Positive Psychology to Realize Your Potential for Lasting Fulfillment. New York: Free Press.

Seligman, M. E. P., y Csikszentmihalyi, M. (2000). Positive
Psychology: An Introduction. American Psychologist, 55, 3-6.

Snyder, C. R. y Lopez, S. J. (Eds.). (2002). Handbook of Positive Psychology. Cambridge, Ma: Oxford University Press.

Strack, F., Argile, M. y Schwartz, N. (Eds.). (1991). Subjective Well-Being. Oxford: Pergamon.

Van Dierendonck, D. (2004). The construct validity of Ryff's Scale of Psychological well-being and its extension with spiritual well-being. Personality and Individual Differences, 36, 629-644.

Van Laar, D, Edwards, J. y Easton, S (2007). The WorkRelated Quality of Life scale for healthcare workers. Journal of Advanced Nursing, 60, 325-333

Veenhoven, R. (1984). Conditions of Happiness. Dordrecht: Reidel.

Veenhoven, R. (1994). El estudio de la satisfacción con la vida. Intervención Psicosocial, 3, 87-116.

Veenhoven, R. (1995). Developments in Satisfaction Research. Social Indicators Research, 37, 1-46.

Veenhoven R (1996). Happy life expectancy: a comprehensive measure of quality-of-life in nations. Social Indicators Research 39, 1-58.

Warr, P. (1987). Work, unemployement and mental health. Oxford: Oxford University Press.

Warr, P. (1990). The measurement of well-being and other aspects of mental health. Journal of Occupational Psychology, 63, 193-210.

White, A. (2007). A Global Projection of Subjective Wellbeing: A Challenge to Positive Psychology? Psychtalk, $56,17-20$.

Manuscrito recibido: 20/05/2010

Revisión recibida: 15/07/2010

Aceptado: 18/07/2010 
Anexo: Escalas e Ítems del Cuestionario de Bienestar Laboral General

\begin{tabular}{|c|c|c|c|c|c|c|c|c|}
\hline \multicolumn{9}{|c|}{$\begin{array}{l}\text { Factor de Bienestar Psicosocial } \\
\text { Escala de Afectos } \\
\text { Instrucciones: Rodee el número elegido }\end{array}$} \\
\hline \multicolumn{9}{|c|}{ Actualmente, en mi trabajo, siento: } \\
\hline Insatisfacción & 1 & 2 & 3 & 4 & 5 & 6 & 7 & Satisfacción \\
\hline Inseguridad & 1 & 2 & 3 & 4 & 5 & 6 & 7 & Seguridad \\
\hline Intranquilidad & 1 & 2 & 3 & 4 & 5 & 6 & 7 & Tranquilidad \\
\hline Impotencia & 1 & 2 & 3 & 4 & 5 & 6 & 7 & Potencia \\
\hline Malestar & 1 & 2 & 3 & 4 & 5 & 6 & 7 & Bienestar \\
\hline Desconfianza & 1 & 2 & 3 & 4 & 5 & 6 & 7 & Confianza \\
\hline Incertidumbre & 1 & 2 & 3 & 4 & 5 & 6 & 7 & Certidumbre \\
\hline Confusión & 1 & 2 & 3 & 4 & 5 & 6 & 7 & Claridad \\
\hline Desesperanza & 1 & 2 & 3 & 4 & 5 & 6 & 7 & Esperanza \\
\hline Dificultad & 1 & 2 & 3 & 4 & 5 & 6 & 7 & Facilidad \\
\hline
\end{tabular}

Escala de Competencias

Instrucciones: Rodee el número elegido

\begin{tabular}{|c|c|c|c|c|c|c|c|c|}
\hline \multicolumn{9}{|c|}{ Actualmente, en mi trabajo, siento: } \\
\hline Insensibilidad & 1 & 2 & 3 & 4 & 5 & 6 & 7 & Sensibilidad \\
\hline Irracionalidad & 1 & 2 & 3 & 4 & 5 & 6 & 7 & Racionalidad \\
\hline Incompetencia & 1 & 2 & 3 & 4 & 5 & 6 & 7 & Competencia \\
\hline Inmoralidad & 1 & 2 & 3 & 4 & 5 & 6 & 7 & Moralidad \\
\hline Maldad & 1 & 2 & 3 & 4 & 5 & 6 & 7 & Bondad \\
\hline Fracaso & 1 & 2 & 3 & 4 & 5 & 6 & 7 & Éxito \\
\hline Incapacidad & 1 & 2 & 3 & 4 & 5 & 6 & 7 & Capacidad \\
\hline Pesimismo & 1 & 2 & 3 & 4 & 5 & 6 & 7 & Optimismo \\
\hline Ineficacia & 1 & 2 & 3 & 4 & 5 & 6 & 7 & Eficacia \\
\hline Inutilidad & 1 & 2 & 3 & 4 & 5 & 6 & 7 & Utilidad \\
\hline
\end{tabular}

Escala de Expectativas

Instrucciones: Rodee el número elegido

\begin{tabular}{lllllllllll}
\hline Está(n) bajando & 1 & 2 & 3 & 4 & 5 & 6 & 7 & Está(n) subiendo
\end{tabular}

En mi trayectoria laboral:

Mi motivación por el trabajo

Mi identificación con los valores de la organización

Mi rendimiento profesional

Mi capacidad de gestión de mi carga de trabajo

La calidad de mis condiciones de trabajo

Mi autoestima profesional

La cordialidad en mi ambiente social de trabajo

La conciliación de mi trabajo con mi vida privada

Mi confianza en mi futuro profesional

Mi calidad de vida laboral

El sentido de mi trabajo

Mi acatamiento de las pautas de la dirección

Mi estado de ánimo laboral

Mis oportunidades de promoción laboral

Mi sensación de seguridad en el trabajo

Mi participación en las decisiones de la organización

Mi satisfacción con el trabajo

Mi realización profesional

El nivel de excelencia de mi organización

Mi eficacia profesional

Mi compromiso con el trabajo

Mis competencias profesionales

$\begin{array}{lllllll}1 & 2 & 3 & 4 & 5 & 6 & 7 \\ 1 & 2 & 3 & 4 & 5 & 6 & 7 \\ 1 & 2 & 3 & 4 & 5 & 6 & 7 \\ 1 & 2 & 3 & 4 & 5 & 6 & 7 \\ 1 & 2 & 3 & 4 & 5 & 6 & 7 \\ 1 & 2 & 3 & 4 & 5 & 6 & 7 \\ 1 & 2 & 3 & 4 & 5 & 6 & 7 \\ 1 & 2 & 3 & 4 & 5 & 6 & 7 \\ 1 & 2 & 3 & 4 & 5 & 6 & 7 \\ 1 & 2 & 3 & 4 & 5 & 6 & 7 \\ 1 & 2 & 3 & 4 & 5 & 6 & 7 \\ 1 & 2 & 3 & 4 & 5 & 6 & 7 \\ 1 & 2 & 3 & 4 & 5 & 6 & 7 \\ 1 & 2 & 3 & 4 & 5 & 6 & 7 \\ 1 & 2 & 3 & 4 & 5 & 6 & 7 \\ 1 & 2 & 3 & 4 & 5 & 6 & 7 \\ 1 & 2 & 3 & 4 & 5 & 6 & 7 \\ 1 & 2 & 3 & 4 & 5 & 6 & 7 \\ 1 & 2 & 3 & 4 & 5 & 6 & 7 \\ 1 & 2 & 3 & 4 & 5 & 6 & 7 \\ 1 & 2 & 3 & 4 & 5 & 6 & 7 \\ 1 & 2 & 3 & 4 & 5 & 6 & 7\end{array}$


Factor de Efectos Colaterales

Escala de Somatización

Instrucciones: Rodee el número elegido

\begin{tabular}{|c|c|c|c|c|c|c|c|c|}
\hline Nunca & 1 & 2 & 3 & 4 & 5 & 6 & 7 & Siempre \\
\hline \multicolumn{9}{|c|}{ Actualmente, por causa de mi trabajo, siento: } \\
\hline Trastornos digestivos & 1 & 2 & 3 & 4 & 5 & 6 & 7 & \\
\hline Dolores de cabeza & 1 & 2 & 3 & 4 & 5 & 6 & 7 & \\
\hline Insomnio & 1 & 2 & 3 & 4 & 5 & 6 & 7 & \\
\hline Dolores de espalda & 1 & 2 & 3 & 4 & 5 & 6 & 7 & \\
\hline Tensiones musculares & 1 & 2 & 3 & 4 & 5 & 6 & 7 & \\
\hline
\end{tabular}

Escala de Desgaste

Instrucciones: Rodee el número elegido

\begin{tabular}{|c|c|c|c|c|c|c|c|c|}
\hline Nunca & 1 & 2 & 3 & 4 & 5 & 6 & 7 & Siempre \\
\hline \multicolumn{9}{|c|}{ Actualmente, por causa de mi trabajo, siento: } \\
\hline Sobrecarga de trabajo & 1 & 2 & 3 & 4 & 5 & 6 & 7 & \\
\hline Desgaste emocional & 1 & 2 & 3 & 4 & 5 & 6 & 7 & \\
\hline Agotamiento físico & 1 & 2 & 3 & 4 & 5 & 6 & 7 & \\
\hline Saturación mental & 1 & 2 & 3 & 4 & 5 & 6 & 7 & \\
\hline
\end{tabular}

Escala de Alienación

Instrucciones: Rodee el número elegido

\begin{tabular}{|c|c|c|c|c|c|c|c|c|}
\hline Nunca & 1 & 2 & 3 & 4 & 5 & 6 & 7 & Siempre \\
\hline \multicolumn{9}{|c|}{ Actualmente, por causa de mi trabajo, siento: } \\
\hline Mal humor & 1 & 2 & 3 & 4 & 5 & 6 & 7 & \\
\hline Baja realización profesional & 1 & 2 & 3 & 4 & 5 & 6 & 7 & \\
\hline Trato despersonalizado & 1 & 2 & 3 & 4 & 5 & 6 & 7 & \\
\hline Frustración & 1 & 2 & 3 & 4 & 5 & 6 & 7 & \\
\hline
\end{tabular}

